the haemagglutinin, in the form seen after ether treatment, does not represent a structure which is present as such in the virus particle before splitting, but a modification of a portion of the outer coat.

The $G$ antigen, on the contrary, is almost certainly present in the form observed after ether splitting. The only question in doubt is the total length of $G$ antigen in the particle, and whether it is in the form of several short pieces, or of one long piece which is disrupted by the ether treatment. The most significant outcome of these findings is that they confirm the prediction of $\mathrm{S}_{\mathrm{CHÄFER}}{ }^{11}$ (1957) that the $G$ antigen would be found to correspond, as a structure, to the whole particle of the smaller RNA viruses. A typical small "spherical" virus (mouse encephalomyelitis (ME) virus) has been sprayed with some $G$ antigen of KP virus, and the two together exemplify the two common forms of arrangement of subunits, i. e. the helical and the polyhedral, e. g. icosahedral or dodecahedral. It is the $\mathrm{G}$ antigen which contains the RNA, and which presumably alone

11 W. SснӥғеR, in: The Nature of Viruses (Ed. A. E. W. WoLstenholme and E. C. P. Millar), p. 100, London 1957. carries the genetic information of the particle, and which has protein subunits symmetrically arranged (a symmetry of form which is not seen in the intact particle).

As far as the von Magnus incomplete forms are concerned, it is clear that the similarity of KP to influenza extends also to the phenomenon of production of incomplete virus by passage of large inocula and that there are similarities between the incomplete forms of the two viruses (Waterson and Horne ${ }^{12}, 1961$; Rott and Schäfer $\left.{ }^{9}, 1960\right)$. The particles of virus $\mathrm{N}$ show much more variation in shape and size than do those of KP, although it is fair to point out that in some passages of KP virus almost as much variation may be found.

We are greatly indebted to Dr. G. F. Meyer and Dr. H. Frank, of the Max-Planck-Institut für Biologie, Tübingen, for assistance with the electron microscopy and to the Deutsche Forschungsgemein. $\mathrm{sch}$ a f $\mathrm{t}$ for financial support. One of us (A.P.W.) is in receipt of a grant from the $\mathrm{Medical} \mathrm{Research}$ Council.

12 A. P. Waterson and R. W. Horne, 1961, to be published.

\title{
Reindarstellung und Charakterisierung des für die Lyse T2-infizierter Zellen verantwortlichen Enzyms
}

\author{
Von W. Weidel und W. Katz \\ Max-Planck-Institut für Biologie, Abt. WeideL, Tübingen \\ (Z. Naturforschg. 16 b, 156-162 [1961]; eingegangen am 11. Januar 1961)
}

\begin{abstract}
T2-infected cells of $E$. coli B synthesize an enzyme whose role it is eventually to lyse the cell from within, whereby the new crop of T2-phage is released from it. Procedures for isolating this enzyme in pure form are described. The enzyme is characterized as a lysozyme. It has a molecular weight of 15200 .
\end{abstract}

Die erste Beobachtung über ein anscheinend nur von phageninfizierten Zellen synthetisiertes, lytisches Enzym stammt von Sertic ${ }^{1}$. Er beschreibt einen „Lysinzonen bildenden“ Phagen Fcz (Wirtsbakterium ist der mucoide E. coli-Stamm $\mathrm{Fb}$ ) und führt die Erscheinung der Zonenbildung, d. h. das Auftreten eines Hofs rings um einen Phagen-Plaque, auf ein lytisches, im Agar diffundibles Agens zurück, das die infizierte Zelle zusammen mit den neugebildeten Phagenteilchen verläßt. Es kann von diesen abgetrennt werden.

\footnotetext{
1 V. Sertic, Zbl. Bakteriol., Parasitenkunde Infektionskrankh., Abt. I, Orig. 110, 125 [1929].

2 A. Gratia, C. R. Séances Soc. Biol. Filiales 126, 418 [1937].
}

Zu ähnlichen Resultaten kam Gratia ${ }^{2}$, der ebenfalls mit einem E. coli-Phagen-System arbeitete.

Humphries ${ }^{3}$ sowie ParK ${ }^{4}$, Adams und ParK ${ }^{5}$ beschreiben ein entsprechendes Enzym in Lysaten verschiedener Stämme von Klebsiella pneumoniae, die mit dem Phagen Kp infiziert waren. Dieses Enzym liegt dabei sowohl an das Phagenteilchen gebunden wie auch in freier Form vor.

Sämtliche bisher aufgeführten Bakterienstämme besitzen eine Polysaccharidkapsel; die Hofbildung ist auf ein Auflösen dieser Kapsel durch das betref-

3 J. C. Humphries, J. Bacteriol. 56, 683 [1948].

4 B. H. PARK, Virology 2, 711 [1956]. 
A. P. Waterson, R. Rott and W. Schäfer, The Structure of Fowl Plaque Virus and iirus N (S. 154)
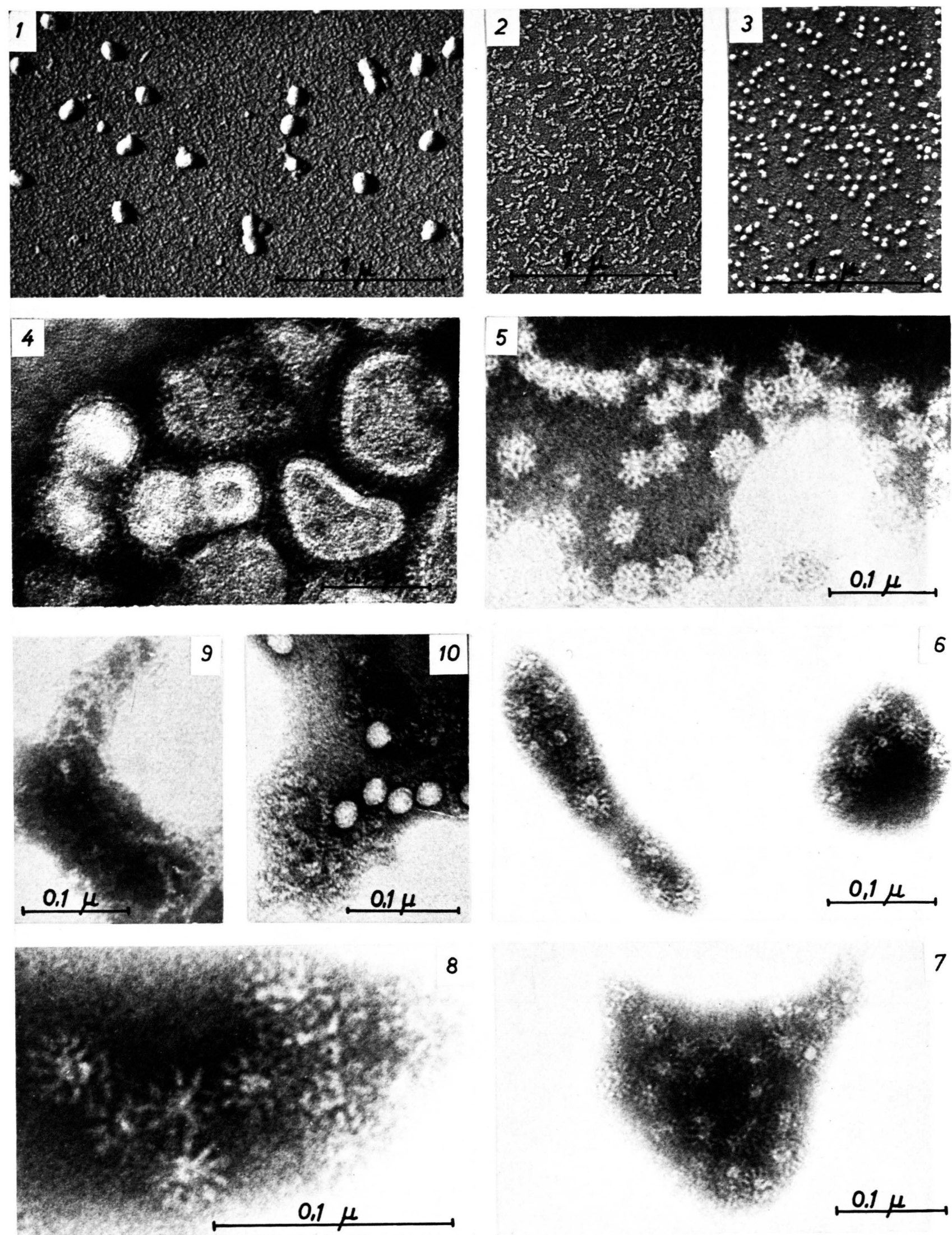

KP virus, platinum-rhodium shadowed: (1) Intact particles, (2) G antigen, (3) Haemagglutinin.

$\mathrm{KP}$ virus, negatively stained: (4) Intact particles, (5, 6, 7, 8) Haemagglutinin, (9) $\mathrm{G}$ antigen, (10) $\mathrm{G}$ antigen with ME virus. 

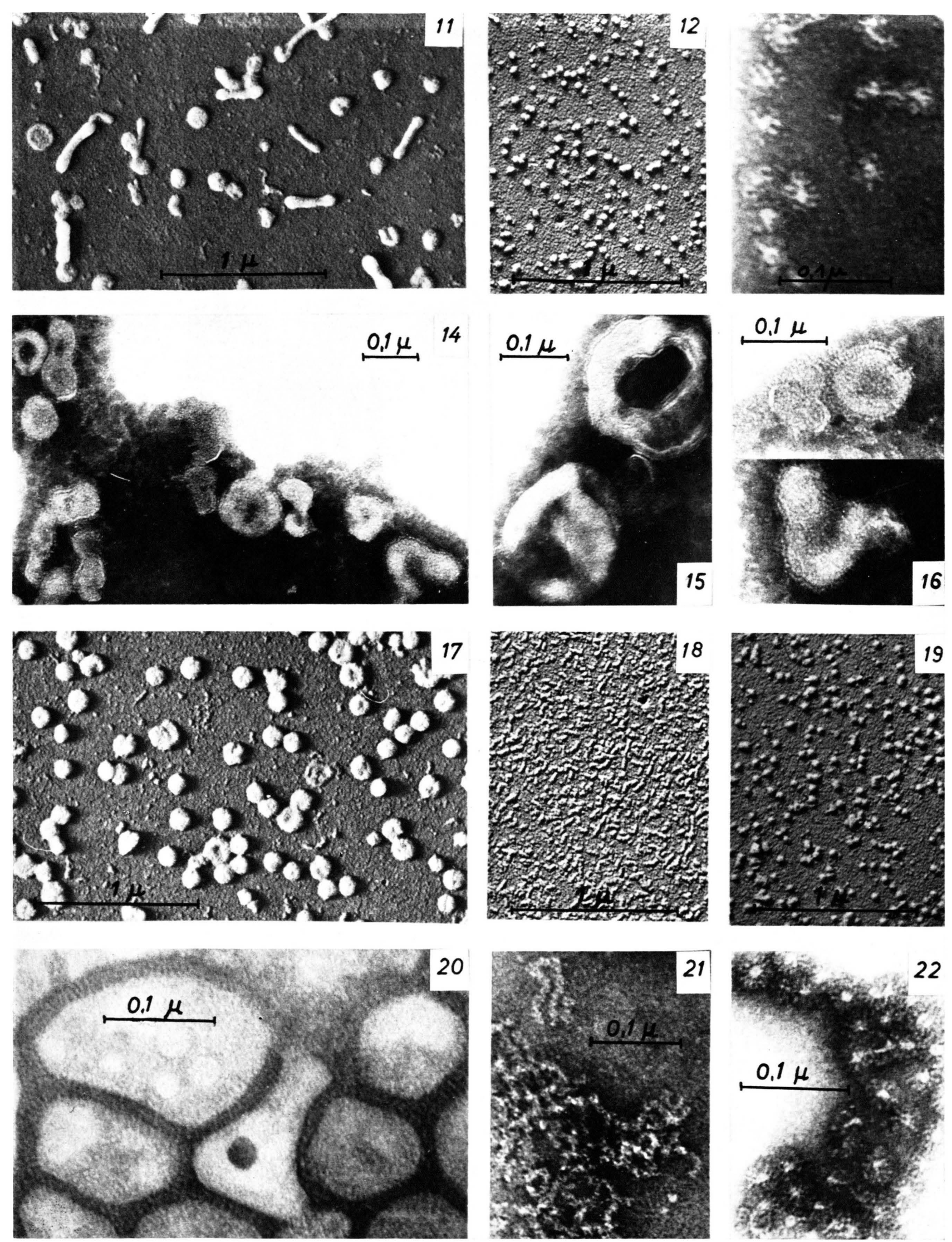

KP virus, incomplete forms: (11) Intact particles, platinum-rhodium shadowed, (12) Haemagglutinin, platinum-rhodium shadowed, (13) Haemagglutinin, negatively stained, (14, 15, 16) intact particles, negatively stained. Virus N, platinum-rhodium shadowed: (17) Intact particles, (18) G antigen, (19) Haemagglutinin. Virus N, negatively stained: (20) Intact particles, (21) $\mathrm{G}$ antigen, (22) Haemagglutinin. 
fende Enzym zurückzuführen. Adams und $\mathrm{PARK}^{5}$ bezeichnen das von ihnen untersuchte Agens generell als Polysaccharid-Depolymerase.

Das Phänomen einer Hofbildung beobachtete MuRPHY ${ }^{6}$ auch bei Bacillus megaterium und dem infizierenden Phagen G. Das Enzym zerstört in diesem Fall die Zellwand des betreffenden Mikroorganismus. Derselbe Autor fand in weiteren Untersuchungen ${ }^{7}$, daß in diesem speziellen System wahrscheinlich zwei lytische Enzyme aufgebaut werden. Das eine ist an die Phagenteilchen gebunden, das andere ist frei. Beide Enzyme greifen zwar dasselbe Substrat, die Zellwand, an, doch unterscheiden sie sich in ihrem serologischen Verhalten und in ihrem Wirkungsoptimum.

Auch von gewissen Phagen der T-Serie und ihrem gemeinsamen Wirt E. coli B liegen solche Befunde vor. So fand Brown ${ }^{8}$, daß chloroformgetötete ColiBakterien durch phagenfreie T6-Lysate lysiert werden.

Koch und Jordan ${ }^{9}$ (s. auch Koch und Dreyer ${ }^{10}$ ) wiesen in T2-Lysaten ein Enzym nach, das nicht an T2-Teilchen gebunden ist, E. coli $\mathrm{B}$ lysiert und isolierte Coli B-Zellwände angreift. Im Rahmen unserer Studien über das an T2-Teilchen gebundene, zellwandzerstörende Enzym ${ }^{11-17}$ nahmen wir uns auch eine nähere Untersuchung dieses in T2-Lysaten in freier Form auftretenden Enzyms vor.

Seine Reindarstellung wird im folgenden beschrieben.

\section{Material und Methoden}

\section{Herstellung von T 2 - Lysaten}

T2-Lysate wurden nach dem von Vielmetter und Schuster ${ }^{18}$ angegebenen Verfahren gewonnen. In einer waagrecht liegenden, mit $180 \mathrm{U} / \mathrm{min}$ rotierenden 20-l-Flasche wurde $E$. coli B jeweils in $500 \mathrm{ml}$ des dort beschriebenen Kulturmediums bei $37^{\circ}$ auf $3 \cdot 10^{9}$ Zellen $/ \mathrm{ml}$ angezüchtet und mit $1,6 \cdot 10^{10} \mathrm{~T} 2 / \mathrm{ml}$ infiziert. Der $p_{\mathrm{H}}$-Wert der Kulturflüssigkeit war kurz vor der T2-Infektion mit 4-m. $\mathrm{NaOH}$ auf 7,3 eingestellt worden. Die infizierte Bakterienkultur wurde 15 Stdn. lang weiterrotiert.

5 M. H. Adams u. B. H. Park, Virology 2, 719 [1956].

6 J. S. Murphy, Virology 4, 563 [1957].

7 J. S. Murphy, Virology 11, 510 [1960].

8 A. Brown, J. Bacteriol. 71, 482 [1956].

9 G. Косн u. E. Jordan, Biochim. biophysica Acta [Amsterdam] 25, 437 [1957].

10 G. Koch u. W. Dreyer, Virology 6, 291 [1958].

11 W. WEIDEL, Z. Naturforschg. 6 b, 251 [1951].

12 W. WEIDEL, Z. Naturforschg. 7 b, 145 [1952].

13 G. Косн u. W. Weidel, Z. Naturforschg. 11 b, 345 [1956].
Nach 6-stdg. Vorbehandlung mit einer Spur Desoxyribonuclease wurden die so erhaltenen Rohlysate, die eine Ausbeute von $4 \cdot 10^{11}$ bis $2 \cdot 10^{12} \mathrm{Phagen} / \mathrm{ml}$ liefern, zur Entfernung von Zelltrümmern für $20 \mathrm{~min}$ bei $4000 \mathrm{~g}$ zentrifugiert; im Anschluß daran wurden die Phagen durch 1-stdg. Zentrifugieren bei $15000 \mathrm{~g}$ sedimentiert.

Der Überstand kann nach abschließendem Zentrifugieren für $45 \mathrm{~min}$ bei $105000 \mathrm{~g}$ ohne weiteres auf freies T2-Enzym aufgearbeitet werden. Das vorgereinigte Lysat sollte mindestens 10 Einheiten Enzym/ml enthalten (Definition s. folgender Absatz).

\section{Quantitative Bestimmung des $\mathrm{Ph}$ a genenzy s}

Der quantitative Test für freies T2-Enzym stützt sich auf eine Beobachtung von Brown ${ }^{8}$, wonach phagenfreie T6-Lysate chloroformgetötete E. coli B-Zellen zu lysieren vermögen.

\section{Herstellung der Zellsuspension}

$300 \mathrm{ml}$ synthetisches Glucosemedium (M9) werden mit $60 \mathrm{ml}$ einer Übernachtkultur von $E$. coli B in M9 angeimpft. Die Suspension wird bei $37^{\circ}$ stark belüftet und das Bakterienwachstum durch Extinktionsmessung bei $650 \mathrm{~m} \mu$ verfolgt. (Beckman Spektralphotometer DU). Die Inkubation wird abgebrochen bei einem Extinktionswert von 0,470 (1-cm-Küvette), was einer Bakteriendichte von $7 \cdot 10^{8}$ Zellen $/ \mathrm{ml}$ entspricht, und der gesamte Ansatz für 15 min in freischwingenden Bechern bei $4200 \mathrm{~g}$ zentrifugiert. Die sedimentierten Bakterien werden bei Zimmertemperatur in insgesamt $100 \mathrm{ml}$ chloroformgesättigtem $m / 15$ Phosphat-Puffer $p_{\mathrm{H}} 7,0$ resuspendiert, sofort im Anschluß daran wie zuvor zentrifugiert, einmal mit chloroformfreiem Phosphat-Puffer auf der Zentrifuge nachgewaschen und schließlich in einer geeigneten Menge Puffer aufgenommen (s. u.).

\section{Ausführung des Tests}

Nach Einstellen der Lichtmarke am Photometer „Eppendorf“ mit Hilfe des Trübungsstandards auf den Skalenwert 50 (Nephelometrie-Filter) wird die Suspension getöteter Bakterien mit soviel $m / 15$ Phosphat-Puffer $p_{\mathrm{H}} 7,0$ verdünnt, da $\beta$ sie, an Stelle des Trübungsstandards, einen Trübungswert von 100 Skalenteilen ergibt (2-cm-Küvette).

In einem Reagenzglas werden $4 \mathrm{ml}$ der so eingestellten Bakteriensuspension mit $0,1 \mathrm{ml}$ zu testender Enzymlösung gemischt und $4 \mathrm{~min}$ bei $37^{\circ}$ inkubiert; nach Abbrechen der Inkubation wird die durch Lyse hervor-

14 W. Weidel u. J. Primosigh, Z. Naturforschg. 12 b, 421 [1957].

15 W. Weidel u. J. Primosigh, J. gen. Microbiol. 18, 513 [1958].

16 W. Weidel, H. Frank u. H. H. Martin, J. gen. Microbiol. 22, 158 [1960].

17 J. Primosigh, H. Pelzer, D. Maass u. W. Weidel, Biochim. biophysica Acta [Amsterdam] 46, 68 [1961].

18 W. Vielmetter u. H. Schuster, Z. Naturforschg. 15 b, 304 [1960]. 
gerufene Trübungsabnahme sofort im Photometer „Eppendorf“" gemessen (2-cm-Küvette).

Es zeigt sich, daß eine nicht mit T2-Enzym versetzte Kontrollsuspension nach $4 \mathrm{~min}$ bei $37^{\circ}$ eine Trübungsabnahme von 100 auf 90 Skalenteile erfährt und daß andererseits, auch mit äußerst hohen Enzymkonzentrationen, nie ein Trübungswert von 47 Skalenteilen unterschritten wird. Innerhalb dieser Grenzen ist die prozentuale Trübungsabnahme bei konstanter Inkubationszeit proportional zur Enzymkonzentration (Abb. 1). Es erschien zweckmäßig, als Maßeinheit (1 E) diejenige Enzymmenge festzulegen, die nach genau 4 min Inkubation bei $37^{\circ}$ den erreichbaren Trübungsminimalwert von 47 Skalenteilen ergibt. Unter diesen Umständen darf bei Enzymtitrationen die im Test eingesetzte Enzymmenge $1 \mathrm{E}$ natürlich niemals ganz erreichen oder gar überschreiten.

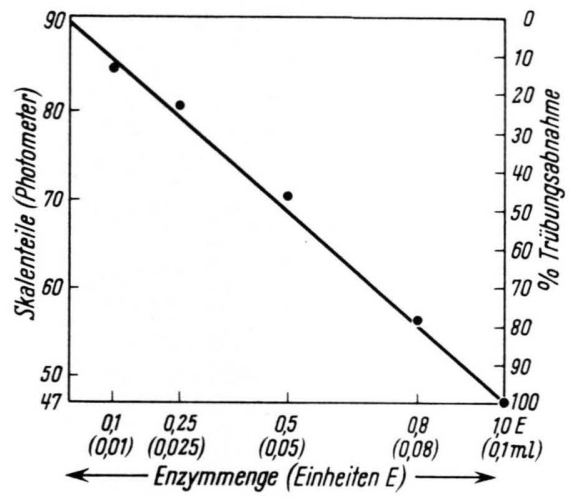

Abb. 1. Lyse chloroformgetöteter Coli-Zellen durch T2-Enzym unter Standardbedingungen (s. Text). Die Gerade gibt die zu erwartende Abhängigkeit des erreichten Lysegrades von der eingesetzten Enzymmenge wieder. Die eingetragenen Punkte wurden mit verschiedenen Volumina einer Enzymlösung gemessen, die genau $10 \mathrm{E} / \mathrm{ml}$ enthielt.

Die für den Test benötigte Bakteriensuspension muß stets frisch sein. Leider verlieren die chloroformgetöteten Zellen sehr schnell, auch in der Kälte, die Fähigkeit zur Lyse, was auch durch Schütteln an der Luft nicht wieder vollständig rückgängig $\mathrm{zu}$ machen ist ${ }^{19}$. Ferner ist stets darauf zu achten, daß die Bakterien das richtige physiologische Alter haben, d. h. die Kultur ist bei Erreichen der angegebenen Zelldichte zu stoppen.

Unter Einhaltung dieser Bedingungen hat man jedoch ein sehr zuverlässiges Verfahren in Händen; die erhaltenen Werte waren stets reproduzierbar.

Lysozym: Sigma Chem. Comp., St. Louis (Mo.).

Micrococcus lysodeikticus: Der Organismus wurde uns, ohne nähere Bezeichnung, von den Behring-Werken, Marburg/Lahn, freundlichst zur Verfügung gestellt, wofür wir auch an dieser Stelle bestens danken möchten.

\section{Ergebnisse}

1. Konzentrierung des Enzyms

Das gelbbraun gefärbte, wie beschrieben durch hochtouriges Zentrifugieren vorgereinigte Rohlysat wird im Rotationsverdampfer unter Wasserstrahlvakuum bei einer Wasserbadtemperatur von 25 bis $30^{\circ}$ auf etwa ${ }^{1} / 10$ seines Volumens eingeengt.

Verluste an Enzymaktivität treten hierbei praktisch nicht in Erscheinung.

Das jetzt tief dunkelbraune, viskose Konzentrat wird 36 Stdn. bei $2^{\circ}$ gegen $m / 50$ Phosphat-Puffer $p_{\mathrm{H}} 7,0$ dialysiert, wobei Schlauchmaterial der Visking Company, Chicago, verwendet werden sollte. Mit anderen Dialysierschlauch-Fabrikaten erlebten wir, offenbar durch irreversible Adsorption von Enzym an das Schlauchmaterial, hohe Verluste an Enzymaktivität. Auf die Dialyse erfolgt ein nochmaliges Einengen der Innenflüssigkeit im Rotationsverdampfer auf das ursprüngliche Konzentratvolumen.

\section{Reinigung des Enzyms}

Das dialysierte Konzentrat wird in der Kälte $\left(2^{\circ}\right)$ unter fortwährendem Rühren tropfenweise mit 1-n. $\mathrm{HCl}$ versetzt. Bei $p_{\mathrm{H}} 4,3$ bricht man die Säurezugabe ab und läßt die Mischung, am besten über Nacht, bei $2^{\circ}$ stehen. Es hat sich dann ein schleimig-flockiger Niederschlag gebildet, der hochtourig abzentrifugiert wird ( $60 \mathrm{~min}$ bei $54500 \mathrm{~g}$ ).

Der Überstand enthält noch die volle Enzymaktivität. Wird die Ausfällung bei $p_{\mathrm{H}} 4,2$ vorgenommen, so werden zwar inaktive Begleitproteine vollständiger niedergeschlagen, doch reißen sie dann schon etwas, bei $p_{\mathrm{H}} 4,1$ bzw. 4,0 sogar sehr viel Enzym mit.

Der saure Überstand wird wiederum 36 Stdn. in der Kälte gegen $m / 50$-Phosphat-Puffer $p_{\mathrm{H}} 7,0$ dialysiert und die Innenflüssigkeit alsdann lyophilisiert.

Zum Trockenrückstand gibt man soviel $m / 50$. Phosphat-Puffer $p_{\mathrm{H}} 7,0$, daß alles eben glatt gelöst ist. Diese Lösung wird nun ein letztes Mal für 48 Stdn., und zwar jetzt gegen $m / 15$-Phosphat-Puffer $p_{\mathrm{H}} 6,0$ dialysiert. Auch bei noch länger ausgedehnter Dialyse verliert die Innenflüssigkeit ihre gelbe Farbe nicht ganz. Im Anschluß daran zentrifugiert man sie für $60 \mathrm{~min}$ bei $105000 \mathrm{~g}$.

Der quantitative Test zeigt, daß dieses bereits erheblich gereinigte Enzymkonzentrat noch 90\% der Aktivität des eingesetzten Rohlysats enthält.

\section{Is olierung des Enzyms}

Zur Reindarstellung des Enzyms wurde die Säulenchromatographie herangezogen. Cellulose in

19 R. Repaske, Biochim. biophysica Acta [Amsterdam] 22, 189 [1956]. 
irgendeiner Form erwies sich als ungeeignetes Adsorbens. Aktives Enzym war nicht mehr davon zu eluieren. In diesem Zusammenhang sei auf unsere Erfahrungen mit gewissen Fabrikaten von (Cellulose-) Dialysierschläuchen hingewiesen (s. o.).

Gute Resultate dagegen lieferte die Anwendung von Kationenaustauschern. Als besonders geeignet befunden wurde das schwach saure Carboxylharz Amberlite CG 50, 100 - 200 mesh (Rohm \& Haas Corp., Philadelphia).

Zur Füllung der Säule benutzt man die durch Schlämmen gewonnenen gröberen Partikel dieses Materials.

Günstige Säulendimensionen: $6,2 \cdot 0,6 \mathrm{~cm}$ für etwa $5 \mathrm{ml}$ gereinigte Enzymlösung, enthaltend zwischen 500 und 3000 Einheiten. Die Vorbehandlung der Säule erfolgt durch Waschen mit dest. Wasser, anschließend mit $n / 10-\mathrm{HCl}$ und wiederum mit Wasser. Durch Behandeln mit $n / 10-\mathrm{NaOH}$ wird das Harz in das Natrium-Salz überführt; nach nochmaligem Waschen mit Wasser wird die Säule mit $m / 15$-Phosphat-Puffer $p_{\mathrm{H}} 6,0$ equilibriert. An eine so vorbereitete Säule wird das Enzym, gelöst im gleichen Puffer, fest gebunden. Oberhalb $p_{\mathrm{H}} 6,5$ findet keine Adsorption mehr statt.

Adsorbiertes Enzym läßt sich durch $m / 2$-Phosphat-Puffer $p_{\mathrm{H}} 7,1$ glatt wieder eluieren. Eine befriedigende Abtrennung von inaktiven Begleitstoffen wird jedoch erst bei Anwendung eines Konzentrations- und $p_{\mathrm{H}^{-}}$Gradienten erzielt. Man erzeugt ihn durch Verwendung von 1-m. Phosphat-Puffer $p_{\mathrm{H}} 7,1$ im Vorratsgefä $\beta(50 \mathrm{ml})$ und $m / 15$-Phosphat-Puffer $p_{\mathrm{H}} 6,0 \mathrm{im}$ Mischgefäß $(50 \mathrm{ml})$.

Die Durchlaufgeschwindigkeit soll nicht größer als 3 Tropfen $/ \mathrm{min}$ sein.

Abb. 2 zeigt das Ergebnis einer Chromatographie von $5 \mathrm{ml}$ durch Säurefällung bei $p_{\mathrm{H}} 4,3$ gereinigten Enzymkonzentrats, enthaltend $100 \mathrm{E} / \mathrm{ml}$. Eluiert wurde in Fraktionen zu je $0,8 \mathrm{ml}$ unter Verwendung des oben angegebenen Puffer-Gradienten.

Die Bestimmung von Gesamtprotein in den Eluatfraktionen erfolgte mit Folin-Ciocalteu-Reagens nach der von Lowry und Mitarbb. ${ }^{20}$ angegebenen Vorschrift (von uns leicht modifiziert) : $0,3 \mathrm{ml}$ jeder Eluatfraktion werden mit $1 \mathrm{ml} \mathrm{Cu-Tartrat-Lösung}$ („Lösung C“) versetzt, gut durchmischt und für 10 min bei Raumtemperatur belassen. Vor Zugabe von $0,1 \mathrm{ml}$ Folin-Reagens, was unter starkem Schüt-

20 O. H. Lowry, N. J. Rosebrough, A. L. FARr u. R. J. RANDaLl, J. biol. Chemistry 193, 265 [1951].

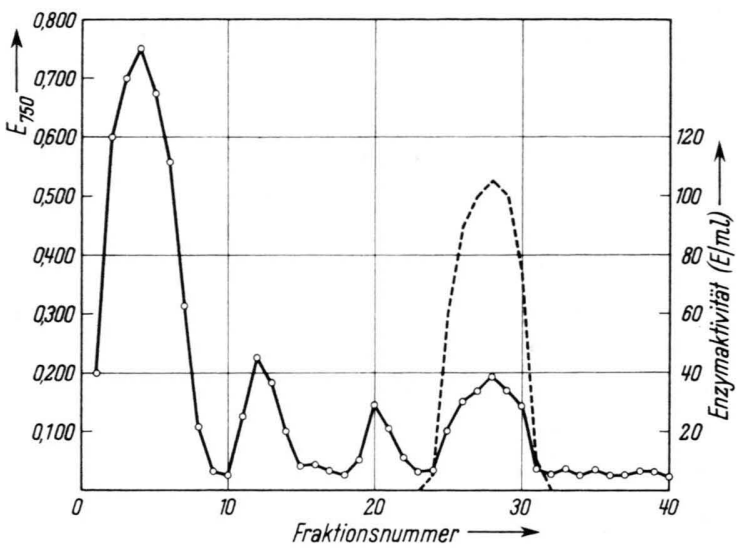

Abb. 2. Chromatographie von T2-Enzym an Amberlite CG 50, (100-200 mesh). - - Enzymaktivität. — Extinktion bei $750 \mathrm{~m} \mu$ nach Umsetzen mit Folin-Ciocalteu-Reagens. Wiedergewonnene Enzymaktivität: 90\% (weitere Angaben: s. Text).

teln vor sich gehen muß, stehen die Proben 2 min bei $70^{\circ} \mathrm{im}$ Wasserbad, da auf diese Weise das Auftreten eines Niederschlags, der sich beim Einpipettieren des Folin-Reagens sonst leicht bildet, umgangen werden kann. Die Reaktionsmischung läßt man dann noch für 30 min bei Raumtemperatur stehen, verdünnt mit $1,4 \mathrm{ml}$ Wasser und bestimmt die Extinktion bei $750 \mathrm{~m} \mu$.

Bei Routine-Aufarbeitungen wurde der beschriebene Enzym-Reinigungsschritt an Amberlite-Harz in fünffach vergrößertem Maßstab durchgeführt. Chromatographiert wurden also jeweils $25 \mathrm{ml}$ des gereinigten Enzymkonzentrats mit ca. $300 \mathrm{E} / \mathrm{ml}$; Säulendimensionen: $11,2 \cdot 1,0 \mathrm{~cm}$; Eluat aufgefangen in 4-ml-Fraktionen.

Zwecks weiterer Konzentrierung des eluierten Enzyms werden sämtliche aktiven Eluatfraktionen gepoolt, $36 \mathrm{Stdn}$. bei $2^{\circ}$ gegen $\mathrm{m} / 50$-Phosphat-Puffer $p_{\mathrm{H}} 7,0$ dialysiert, eingefroren und lyophilisiert. Der Rückstand wird mit wenigen $\mathrm{ml} m / 50$-PhosphatPuffer $p_{\mathrm{H}}$ 7,0 eben in Lösung gebracht und das Konzentrat erneut $48 \mathrm{Stdn}$. lang in der Kälte gegen $m / 15$-Phosphat-Puffer $p_{\mathrm{H}} 7,0$ dialysiert. Das so erhaltene Präparat zentrifugiert man zur Entfernung eingeschleppter Verunreinigungen (Abrieb vom Austauscherharz bzw. Dialysierschlauch) $30 \mathrm{~min}$ lang bei $105000 \mathrm{~g}$.

Diese hochgereinigte Enzymlösung enthält jetzt pro Enzymeinheit 0,32 $\mu \mathrm{g}$ Stickstoff ( $\mathrm{K} \mathrm{j} \mathrm{e} \mathrm{ld} \mathrm{a} \mathrm{h} \mathrm{l).}$

Eine Probesedimentation mit diesem Präparat $(800 \mathrm{E}=256 \mu \mathrm{g} \mathrm{N} / \mathrm{ml})$ in der analytischen Ultrazentrifuge (Spinco, Modell E) ergab im Sedimenta- 
tionsdiagramm einen einzelnen, noch etwas asymmetrischen Gipfel. Durch Wiederholung der Austauschchromatographie ließ sich dieser Mangel beseitigen, das Enzym also in anscheinend völlig reiner Form gewinnen.

$3 \mathrm{ml}$ Enzymlösung der oben angegebenen Konzentration und Reinheitsstufe wurden $48 \mathrm{Stdn}$. gegen $m / 15$ Phosphat-Puffer $p_{\mathrm{H}} 6,0$ dialysiert $\left(2^{\circ}\right)$ und anschließend, wie beschrieben, chromatographiert, jedoch unter Verzicht auf Gradientenelution.

Nach Aufziehen der Enzymlösung auf eine Säule von $8,0 \cdot 0,6 \mathrm{~cm}$ wurde mit $15 \mathrm{ml} \mathrm{m} / 15$-PhosphatPuffer $p_{\mathrm{H}} 6,0$ nachgewaschen und mit etwa 12 bis $15 \mathrm{ml}$ 0,33-m. Phosphat-Puffer $p_{\mathrm{H}} 7,1$ eluiert.

Die Ausbeute an wiedergewonnener Enzymaktivität betrug 80 Prozent. Das wiederum in $m / 15$ Phosphat-Puffer überführte Enzym wurde daraufhin erneut einer Reinheitsprüfung unterzogen. In der analytischen Ultrazentrifuge lieferte es jetzt $(0,29 \mu \mathrm{g} \mathrm{N} / \mathrm{E} ; 1400 \mathrm{E}=406 \mu \mathrm{g} \mathrm{N} / \mathrm{ml})$ einen symmetrischen Gipfel mit einem Sedimentationskoeffizienten $S_{20}=1,76 \cdot 10^{-13}$ Sekunde.

4. Eigenschaften des T2-Enzyms

\section{a) Allgemeines}

T2-Enzym ist ein relativ niedermolekulares Protein vom Mol.-Gew. $15200 \pm 10 \%$, jedoch nicht dialysabel, entgegen einer Behauptung von Косн und JORDAN ${ }^{9}$.

Es verliert seine Aktivität zum größten Teil bei Überführung in ein ionenfreies Milieu. Rechromatographiertes Enzym $(500 \mathrm{E} / \mathrm{ml})$ in $m / 15$-PhosphatPuffer $p_{\mathrm{H}} 7,0$ enthielt nach 72 -stdg. Dialyse gegen dest. Wasser bei $2^{\circ}$ nur noch $10 \%$ seiner ursprünglichen Aktivität. Bei erneuter Dialyse dieses stark inaktivierten Präparates ( $72 \mathrm{Stdn}$. bei $2^{\circ}$ ) gegen $m / 15$-Phosphat-Puffer $p_{\mathrm{H}} 7,0$ stellte sich jedoch die volle Ursprungsaktivität wieder ein.

Man kann das Enzym, ohne es zu schädigen, in physiologischer Kochsalzlösung, m/20-Ammoniumacetat oder $m / 50$ bis 1- $m$. Phosphat-Puffer gelöst aufheben. Es ist also in bezug auf die qualitative und quantitative Zusammensetzung des umgebenden Ionenmilieus nicht besonders anspruchsvoll. Zusätze wie Rohrzucker, Äthylendiamintetraessigsäure (EDTA) oder Trishydroxymethylaminomethan (Tris) sind ebenfalls harmlos.

Von größerem Einfluß dagegen ist die Wasserstoffionenkonzentration. Während sich das Enzym gegenüber einem niedrigen $p_{\mathrm{H}}$-Wert als ausgespro- chen stabil erweist, macht sich bei $p_{\mathrm{H}}>8$ eine erhebliche Instabilität bemerkbar. Um sein Verhalten unter extremen Bedingungen zu demonstrieren, sei folgender Versuch hier angeführt: Zwei Proben desselben Enzympräparates wurden auf $p_{\mathrm{H}} 1,2 \mathrm{bzw}$. 12,8 gebracht und 60 min bei Raumtemperatur belassen. Nach Rückneutralisation auf $p_{\mathrm{H}}$ 7,0 war die Aktivität der „sauren“ Probe noch unverändert, die der „alkalischen“ dagegen zu über $50 \%$ zerstört.

Vollkommene Zerstörung des Enzyms wird auch durch Trypsinbehandlung erzielt. T2-Enzym $(80 \mathrm{E} / \mathrm{ml})$, mit $100 \mu \mathrm{g} / \mathrm{ml}$ krist. Trypsin in $m / 15$ Phosphat-Puffer bei $p_{\mathrm{H}} 7,8$ und $37^{\circ}$ inkubiert, be$\mathrm{sa}$ nach einer Stde. Inkubationszeit noch die Hälfte seiner ursprünglichen Aktivität, nach $2 \mathrm{Stdn}$. noch $10 \%$, nach $3 \frac{1}{2}$ Stdn. war keine Aktivität mehr nachweisbar. Eine Kontroll-Lösung desselben EnzymPräparates, beim gleichen $p_{\mathrm{H}}$-Wert, jedoch ohne Trypsin-Zusatz, inkubiert, besaß nach $3^{1 / 2}$ Stdn. noch eine Aktivität von $70 \mathrm{E} / \mathrm{ml}$ (alkalisches Milieu, s. o.).

Das Wirkungsoptimum des T2-Enzyms liegt zwischen $p_{\mathrm{H}} 6$ und 7. In diesem Bereich sind keine Unterschiede in der Lyserate chloroformgetöteter Bakterien festzustellen.

Wird der quantitative Test bei $p_{\mathrm{H}} 5,7$ durchgeführt, sinkt die Lyserate auf ca. $80 \%$, bei $p_{\mathrm{H}} 5,5$ auf $40 \%$ und bei $p_{\mathrm{H}} 5,2$ auf $10-20 \%$ ab, während sich bei $p_{\mathrm{H}} 7,5$ und 8,0 Werte von $60 \%$ bzw. $20 \%$ ergeben.

Durch zweimalige Chromatographie gereinigtes Enzym läßt sich durch Ammonsulfat ohne größeren Aktivitätsverlust umfällen. Aus $2 \mathrm{ml}$ einer derart gereinigten Enzymlösung $(500 \mathrm{E} / \mathrm{ml})$ in $m / 15$ Phosphat-Puffer $p_{\mathrm{H}} 7,0$ ließ sich das Enzym bei $-2^{\circ}$ durch tropfenweise Zugabe einer gesättigten $\left(\mathrm{NH}_{4}\right)_{2} \mathrm{SO}_{4}$-Lösung bei $80 \%$ Sättigung präzipitieren. Der Ansatz blieb über Nacht bei $+2^{\circ}$ stehen und wurde anschließend für $30 \mathrm{~min}$ bei $5000 \mathrm{~g}$ zentrifugiert. Das in $2 \mathrm{ml} m / 15$-Phosphat-Puffer $p_{\mathrm{H}} 7,0$ wieder aufgelöste Sediment ergab im Test ca. $80 \%$ der Ausgangsaktivität.

Ammonsulfat-Fällung von nicht-chromatographiertem Enzym, das durch Säurefällung gereinigt wurde, zieht hohe Aktivitätsverluste (80\%) nach sich.

\section{b) Klassifizierung}

In einer anderen Arbeit konnte das an T2-Teilchen gebundene, lytische Enzym, entsprechend seiner 
Substratspezifität, als ein „Lysozym“ charakterisiert werden ${ }^{17}$. Aus verschiedenen Gründen liegt es nahe zu vermuten, daß das hier beschriebene, in T2-Lysaten frei vorkommende Enzym ebenfalls ein Lysozym ist und zumindest in dieser Hinsicht mit dem gebundenen T2-Enzym vollkommen übereinstimmt ${ }^{21}$. Eine entsprechende Vermutung äußerten auch $\mathrm{KocH}$ und Dreyer ${ }^{10}$.

Lysozym setzt aus Zellwänden von $E$. coli B eine komplexe Mischung von chemisch eng verwandten Spaltprodukten frei, die sich papierchromatogra-

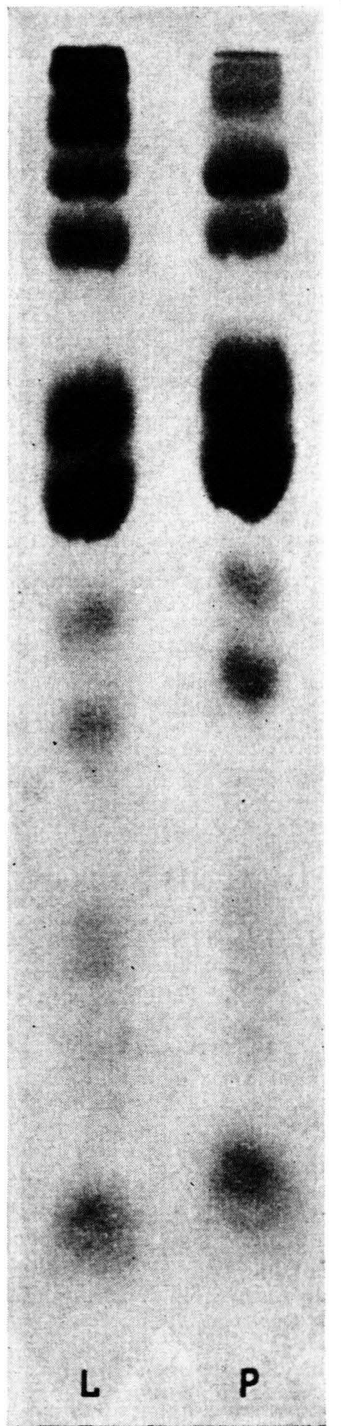

Abb. 3. Parallelchromatogramm enzymatisch abgespaltener Komponenten aus E. coli B-Zellwänden nach 24 Stdn. Inkubation $\left(37^{\circ}\right)$ mit $250 \mu \mathrm{g}$ Lysozym (L) bzw. $250 \mu \mathrm{g}$ freiem

T2-Enzym/ml Inkubationsansatz (P). (Weitere Versuchsbedingungen: s. l. c. $\left.{ }^{17}\right)$. phisch trennen lassen und so ein sehr charakteristisches "Spektrum" liefern ${ }^{17}$.

Abb. 3 zeigt, daß das freie T2-Enzym aus E. coli B-Zellwänden dieselbe Serie von Spaltstücken herausholt wie Lysozym. Damit bestätigen sich früher geäußerte Vermutungen: es ist ebenfalls ein Lysozym.

Weitere, zweifellos gröbere Parallelen zwischen der Wirkungsweise beider Enzyme kann man auch in Folgendem sehen: Lysozym lysiert chloroformgetötete Coli-Zellen in $m / 15$-Phosphat-Puffer $p_{\mathrm{H}} 7,0$, und andererseits lysiert freies T2-Enzym das klassische „Substrat" für Lysozym, d. h. Zellen von Micrococcus lysodeikticus.

Der erstere Befund erlaubt einen Aktivitätsvergleich zwischen beiden Enzymen. Eine Lösung von freiem T2-Enzym mit einem Stickstoffgehalt von $0,29 \mu \mathrm{g} \mathrm{N} / \mathrm{E}$ hat in unserem quantitativen Test dieselbe Aktivität wie eine Lysozym-Lösung mit einem Stickstoffgehalt von $0,35 \mu \mathrm{g}$ N/E. Damit läßt sich sicher sagen, daß beide Enzyme etwa die gleiche spezifische Aktivität (Aktivität/mg Protein) haben.

Nach Koch und Jordan ${ }^{9}$ sowie Koch und Dreyer $^{10}$ soll das freie T2-Enzym übrigens auch intakte $E$. coli B-Zellen ohne weiteres zu lysieren vermögen. Dieser Befund konnte nicht bestätigt werden. Suspensionen gewaschener Bakterien in $m / 15$ Phosphat-Puffer $p_{\mathrm{H}} 7,0$ oder in physiologischer Kochsalzlösung zeigten nach Inkubation bei $37^{\circ}$ mit T2-Enzym bis zu Konzentrationen von $500 \mathrm{E} / \mathrm{ml}$ niemals eine Abnahme ihrer optischen Dichte. Zur Lyse lebender E. coli B-Zellen bedarf es, wie bei Lysozym ${ }^{19}$, der Anwendung von Adjuvantien im Medium: Tris-Puffer, $p_{\mathrm{H}} 8$ und EDTA. Dann sind gleiche Gewichtsmengen Lysozym oder T2-Enzym gleich wirksam.

Micrococcus lysodeikticus dagegen wird ohne Adjuvantien, d. h. in $m / 15$-Phosphat-Puffer $p_{\mathrm{H}} 7,0$, durch T2-Enzym mit derselben Geschwindigkeit

\begin{tabular}{|c|c|c|c|}
\hline $\begin{array}{c}\text { Inkub.-Dauer } \\
\text { [min] }\end{array}$ & T2-Enzym & Lysozym & Puffer \\
\hline 0 & 0,350 & 0,350 & 0,350 \\
30 & 0,285 & 0,275 & 0,350 \\
90 & 0,195 & 0,180 & 0,345 \\
180 & 0,180 & 0,165 & 0,345 \\
\hline
\end{tabular}

Tab. 1. Abnahme der optischen Dichte (bei $650 \mathrm{~m} \mu$ ) einer Suspension von M.lysodeikticus während der Inkubation $\left(37^{\circ}\right)$ mit T2-Enzym bzw. Lysozym (je $50 \mu \mathrm{g} / \mathrm{ml}$ Phosphat-Puffer $p_{\mathrm{H}} 7,0$ ).

21 W. WeIdel, Annu. Rev. Microbiol. 12, 27 [1958]. 
lysiert wie durch Lysozym in entsprechender Konzentration (Tab. 1).

\section{Diskussion}

Die Reindarstellung des von $\mathrm{T}_{2}$-infizierten $E$. coli B-Zellen in beträchtlicher Menge synthetisierten, lytischen Enzyms erweist sich als technisch relativ einfach, so daß das Sammeln genügender Mengen freien Enzyms für weitere Untersuchungen kein Problem ist.

In bezug auf die Ergiebigkeit des Ausgangsmaterials gestaltet sich die Lage erheblich ungünstiger bei der Gewinnung des entsprechenden, an T2-Teilchen gebundenen Enzyms. Dessen Präparierung verläuft zwar im wesentlichen nach dem hier beschriebenen Verfahren, das tatsächlich zunächst hierfür ausgearbeitet wurde, und auch seine Freisetzung aus T2-Teilchen ist nicht schwierig ${ }^{14,15}$, aber die erzielten Ausbeuten reichten noch nicht aus, um einen wirklich detaillierten Vergleich mit dem freien Enzym zu erlauben.

Wir konnten zwar zeigen, daß beide Enzyme das gleiche Substrat, eine bestimmte Schicht der ColiZellwand ${ }^{16}$, hydrolytisch angreifen und es in die gleichen Bestandteile zerlegen ${ }^{17}$, daß sie in dieser Beziehung wiederum mit Lysozym aus Eiklar übereinstimmen und somit ebenfalls als Lysozyme zu bezeichnen sind. Auch die besondere Rolle, die jedes von ihnen bei der Ingangsetzung bzw. Beendigung eines Vermehrungszyklus des T2-Phagen zu spielen hat, wurde verständlich gemacht ${ }^{21}$. Ein noch eingehenderer, insbesondere struktureller Vergleich wäre aber erwünscht, um weitere, sehr interessante Fragen beantworten zu können.

Um nur eine davon herauszugreifen: man wüßte gern, ob das in T2-Teilchen eingebaute, lytische Enzym einem großen, intrazellulären Pool von Enzym entnommen wird, dessen unverbrauchter Überschuß die Lyse der Zelle besorgt und dann als freies Enzym im Lysat erscheint, oder ob das teilchengebundene Enzym vielleicht keineswegs aus einem solchen Pool geschöpft, sondern nur in situ synthetisiert werden kann, d. h. an Ort und Stelle dort, wo jedes T2-Teilchen für sich den sogenannten Reifungsprozeß durchläuft. Dies wäre zwar nicht sehr ökonomisch, weil dann tatsächlich zwei unabhängige Synthesemechanismen von der infizierten Zelle aufgebaut werden müßten - einer für freies und einer für gebundenes Enzym -, obwohl im Grunde nur ein Enzym gebraucht wird, soweit das anzugreifende Substrat in Betracht steht. Womöglich ist aber nur dieser unökonomische Weg gangbar, und dann müßte man erwarten, daß die beiden Enzyme zwar funktionell, aber nicht strukturell übereinstimmen. Die schon erwähnten Befunde von Murphy ${ }^{6}$ an einem anderen System scheinen uns in diesem Sinne zu deuten.

Herrn Dr. K. Wagner sind wir für die Durchführung und Auswertung der Ultrazentrifugation, Frl. K. VoLL für ihre zuverlässige Hilfe bei der Herstellung von T2Lysaten zu großem Dank verpflichtet.

\title{
Uber die Geschwindigkeit der Protein-Synthese in kernlosen und kernhaltigen Zellen von Acetabularia*
}

\author{
Von H. Clauss und G. Werz \\ Aus dem Max-Planck-Institut für Meeresbiologie, Wilhelmshaven (Abt. HäмmerLing) \\ (Z. Naturforschg. 16 b, 162-165 [1961] ; eingegangen am 23. Dezember 1960)

\begin{abstract}
Comparing nucleate and enucleated cells of Acetabularia mediterranea and A. crenulata, the question was reexamined whether or not there is a temporary stimulation of protein synthesis in enucleated cells as reported by BRACHET and coworkers. The data presented here clearly show that there is no higher protein synthesis in enucleated cells than in nucleate ones: The rate of protein formation of enucleated cells is at most equal to that of nucleate cells during the first time following enucleation. It is concluded, that there is no inhibitory effect of the nucleus on protein synthesis.
\end{abstract}

Aus ihren Proteinbestimmungen an kernhaltigen und kernlosen Zellteilen von Acetabularia mediterranea schlossen Brachet und Mitarbb. ${ }^{1}$ (Abb. 9),

\footnotetext{
* Mit Unterstützung durch die Deutsche For schungsgemeinsch a ft.
}

daß bei kernlosen Zellteilen innerhalb der ersten 18 Tage nach ihrer Herstellung eine stärkere Proteinvermehrung erfolge als bei kernhaltigen, der

\footnotetext{
1 J. Brachet, H. Chantrenne u. F. Vanderhaeghe, Biochim. biophysica Acta [Amsterdam] 18,544 [1955], Abb. 9.
} 